\title{
A Low Power 5Gb/s Transimpedance Amplifier with Dual Feedback Technique
}

\author{
I-Hsin Wang, Chung-Shun Liu, and Shen-Iuan Liu \\ Department of Electrical Engineering \& Graduate Institute of Electronics Engineering \\ National Taiwan University, Taipei 10617, Taiwan
}

Abstract-This paper presents the design and implementation of a $5 \mathrm{~Gb} / \mathrm{s}$ transimpedance amplifier (TIA) with dual shunt-shunt feedback technique to enhance the circuit performance. Measured bandwidth is $4.9 \mathrm{GHz}$ and measured transimpedance gain is $60 \mathrm{~dB} \Omega$. The chip consumes only $8 \mathrm{~mW}$ at $1.8 \mathrm{~V}$ supply voltage and occupies $650 \mu \mathrm{m} \times 500 \mu \mathrm{m}$ in a $0.18 \mu \mathrm{m}$ CMOS process.

I. Introduction

The recent growth in the optical fiber communication industry has resulted in high demand for low-cost high-performance optical front ends. One of the critical components of the optical fiber receiver front end is the transimpedance amplifier. A TIA requires high transimpedance gain, high bandwidth, low input impedance, and low power consumption. However, the power consumption trade off with gain and bandwidth product. In this paper, the proposed dual shunt-shunt feedback network used to improve this trade off.

\section{TIA architecture}

The complete architecture of the proposed TIA is depicted in Fig. 1. It consists of a bias network, core amplifier, and the output buffer.

\section{A. Core amplifier}

The core amplifier, depicted in Fig. 1, has to provide a sufficient voltage gain so as to increase the TIA's bandwidth and make its $Z_{21}$ very close to $R_{F}$. In this amplifier, the cascode common source amplifier can eliminate the Miller capacitance effect. However, large capacitance due to photodiode's parasitic effect makes the design more difficult. In order to minimize the power dissipation, the inductive peaking technique $[1]$ is utilized in this circuit, since it does not occupy additional voltage headroom and can improve the speed effectively.

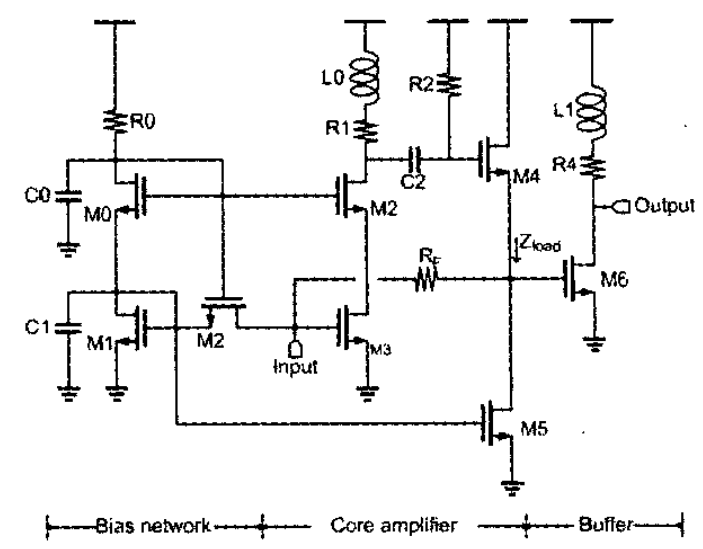

Fig. 1 The proposed TIA

One of the dual shunt-shunt feedback networks in the proposed core amplifier is composed of the MOS transistors $M_{2} \sim M_{4}$, AC couple capacitor $C_{2}$, and feedback resistor $R_{F}$. Assume the amplifier exhibits a mid band gain $A_{0}$ and one pole at $\omega_{0}$, then its transfer function is given by Equation (1).

$$
A(s)=\frac{A_{0}}{1+s / \omega_{0}}
$$

The transfer function of a TIA can be given as

$$
\frac{V_{o u t}}{I_{\text {in }}}(s)=-\frac{A_{0} R_{F}}{\frac{R_{F} C_{P D}}{\omega_{0}} s^{2}+\left(R_{F} C_{P D}+\frac{1}{\omega_{0}}\right) s+A_{0}+1}
$$

where it is assumed that $A_{0}>1$, and $C_{P D}$ is the photo diode capacitance. Usually, it is a typical second order system. The denominator of a second-order transfer function is expressed as $s^{2}+2 \xi \omega_{n} s+\omega_{n}{ }^{2}$, then $\xi$, the "damping factor," must be equal to $\sqrt{2} / 2$ for critical damping. Rewriting (2), we have

$$
\frac{V_{\text {out }}}{I_{\text {in }}}(s)=-\frac{A_{0} R_{F}}{s^{2}+\frac{R_{F} C_{P D}+1 / \omega_{0}}{R_{F} C_{P D} / \omega_{0}} s+\frac{\left(A_{0}+1\right) \omega_{0}}{R_{F} C_{P D}}}
$$

When $\xi=\sqrt{2} / 2$, then

$$
\omega_{0} \approx \frac{2 A_{0}}{R_{F} C_{P D}}
$$

Thus, the $-3 \mathrm{~dB}$ bandwidth of the core amplifier must 
be chosen equal to twice the closed-loop bandwidth of the first-order TIA to ensure a critical-damped response. With the above condition for critical damping, the $-3 \mathrm{~dB}$ bandwidth of the TIA is

$$
\omega_{n}=\sqrt{\frac{\left(A_{0}+1\right) \omega_{0}}{R_{F} C_{P D}}} \approx \frac{\sqrt{2} A_{0}}{R_{F} C_{P D}}
$$

From equation (2) and (5), we have to choice $R_{F}$ carefully to trade off between transimpedance gain and bandwidth. In our TIA, we take the $R_{F}$ equals $1 \mathrm{k} \Omega$ for $60 \mathrm{~dB}$ transimpedance gain in bandwidth better than $5 \mathrm{GHz}$ without photo diode capacitance loading.

Between the first stage and the source follower, a capacitor $(0.8 \mathrm{p})$ for ac coupling is connected. This is because we need large bias current in the first stage to provide sufficient voltage gain but will results in a lower dc level. If we apply dc couple between the two stages, the output dc level of the source follower will be too low to turn on the buffer transistor. The bias resistor of the source follower is realized by a PMOS operating in the triode region.

For the cascaded circuits, they usually have a pole at each stage output due to loading circuit. In our TIA circuit, there is a pole in the output of the core amplifier. If the loading impedance $Z_{\text {load }}$ is large, this pole will become dominated and decrease circuit bandwidth. In order to eliminate this effect on core amplifier's bandwidth, a second shunt-shunt feedback network is incorporated in the output of the core amplifier. This feedback network is composed of the MOS transistors $M_{I} \sim M_{2}$ and $M_{5}$, resistor $R_{F}$, and capacitor $C_{l}$. The transistor $M_{2}$ is operated in triode region. Assume the capacitor $C_{l}$ is larger than the parasitic capacitance of the MOS transistors, $C_{g s}$ and $C_{g d}$. The on resistance of $M_{2}$ is $R_{o n 2}$, and $r_{o s}$ is the output resistance of $M_{5}$. The loading impedance of the core amplifier can be written as

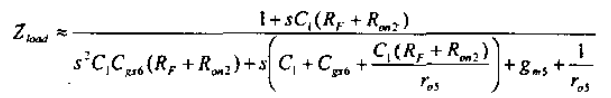

It induces a zero and if one properly chooses the value of $C_{l}$ and $R_{o n 2}$, the bandwidth can be extended by canceling a pair of zero and pole. On the other hand, capacitor $C_{l}$ short the $\mathrm{AC}$ signal to ground, the input signal will not go through transistor $M_{5}$ to the output. By this topology the bandwidth of the core amplifier can be enhanced without increasing power consumption.

\section{B. Noise Optimization}

To optimize the input-referred noise of a TIA, one must very carefully choose the size of the input device since it contributes most of the noise [2]. Consider the simplified noise model shown in Fig. 2.

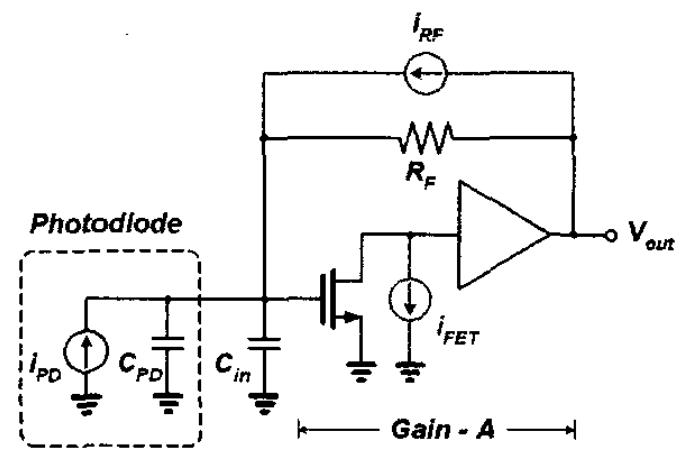

Fig. 2 Equivalent model for optimizing the input noise.

There are two main noise sources: the thermal noise current of the feedback resistor and the channel thermal noise current of input transistor. The expression for the input-referred current density is given by

$$
\overline{i_{n, \text { in }}^{2}}=\frac{4 k T}{R_{F}}+\frac{\overline{V^{2}{ }_{n A, \text { out }}}}{R_{T}(s)^{2}}
$$

Where $V_{n A, \text { out }}$ represents the output noise voltage due to the core amplifier and we have

$$
V_{n A . o u t}=\frac{-A\left(s R_{F} C_{D}+1\right)}{s R_{F} C_{D}+A+1} 4 k T \gamma \frac{1}{\sqrt{g_{m}}}
$$

Substituting equation (8) into (7), we can derive the complete form of input-referred noise

$\overline{i_{n, i n}^{2}}=4 k T\left[\frac{1}{R_{F}}+\frac{\gamma}{{R_{F}{ }^{2} g_{m}}_{m}}+\gamma \omega^{2}\left(\frac{C_{P D}}{\sqrt{g_{m}}}+\frac{\sqrt{g_{m}}}{\omega_{r}}\right)^{2}\right]$

In (9), the second term can be neglected compared 
with the first term, where $\omega_{T}$, the unit gain frequency of a transistor, equals to $\mathrm{gm} /\left(C_{g s}+C_{g d}\right)$. To optimize the spectral density of input-referred noise current, we should make $g_{m}=C_{P D} \cdot \omega_{T}$ or to choose the input transistor size such that $C_{g s}+C_{g d}=$ $C_{P D}$. Although the above example tells us that when $C_{g s}+C_{g d}=C_{P D}$, the noise will be reduced to the minimum level. The transistor size will be quite large and makes it difficult to reach a high bandwidth. We have to choice the core amplifier architecture and circuit layout carefully for this trade off.

\section{Output buffer}

In order to drive the $50 \Omega$ impedance of the oscilloscope, the output buffer should have a high current driving ability. In some recent researches, the common-source type amplifier has become a better choice. First, the output dc level is determined independent of the input de level thus will not suffer from smaller voltage headroom. Second, the output matching can be achieved by simply place a $50 \Omega$ resistor or two $100 \Omega$ resistor in parallel.

\section{Experiment results}

This work has been fabricated in $0.18 \mu \mathrm{m} 1 \mathrm{P} 6 \mathrm{M}$ CMOS process and the die photo is given in Fig. 3 . To measure the frequency domain performance, the TIA chip is probe-tested with network analyzer. The measured transimpedance gain, $Z_{21}$ is given in Fig. 4 . The measured $-3 \mathrm{~dB}$ bandwidth is $4.9 \mathrm{GHz}$ and the midband transimpedance gain is $60 \mathrm{~dB} \Omega$. The measured eye diagrams are given in Fig.5. The eye diagrams at $2.5 \mathrm{Gbps}, 3.125 \mathrm{Gbps}$ and $5 \mathrm{Gbps}$ when the input equivalent current equals $30 \mathrm{uA}$ respectively. Not only the first eye diagram fits in with the OC-48 mask very well but also widely open at the data rate of $5 \mathrm{~Gb} / \mathrm{s}$.

IV. Conclusion

In this work, a $1.8 \mathrm{~V}, 5 \mathrm{Gbps}$ low power transimpedance amplifier using dual shunt-shunt feedback technique is implemented on a standard 0.18 um CMOS process. By this way, the circuit bandwidth can be enhanced and only has $8 \mathrm{~mW}$ power consumption. The characteristics of this circuit are listed in table $\mathrm{I}$.

Table I Performance summary

\begin{tabular}{|c|c|}
\hline Process & $0.18 \mathrm{um}$ CMOS \\
\hline Supply Voltage & $1.8 \mathrm{~V}$ \\
\hline Speed (bit/s) & $5 \mathrm{G}$ \\
\hline Trans. Gain $(\Omega)$ & $1 \mathrm{k}$ \\
\hline Sensitivity & $20 \mathrm{uA}$ \\
\hline Power Dissipation $(\mathrm{mW})$ & 8 \\
\hline Input-referred noise current & $9 \mathrm{pA} / \sqrt{\mathrm{Hz}}$ \\
\hline Chip Area $\left(\mu \mathrm{m}^{2}\right)$ & $650 \times 500$ \\
\hline
\end{tabular}

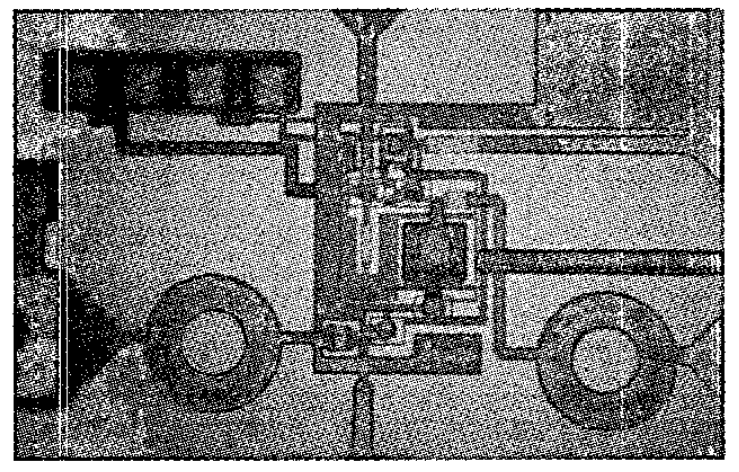

Fig.3 Die photograph of the TIA

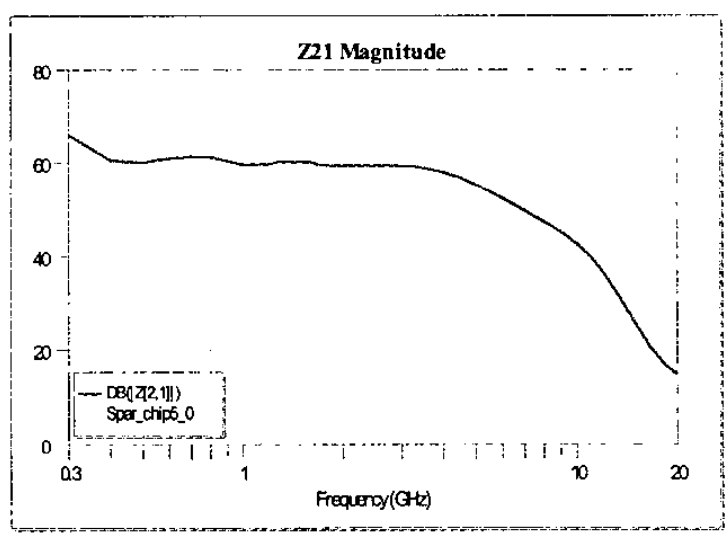

Fig.4 The measured transimpedance gain 


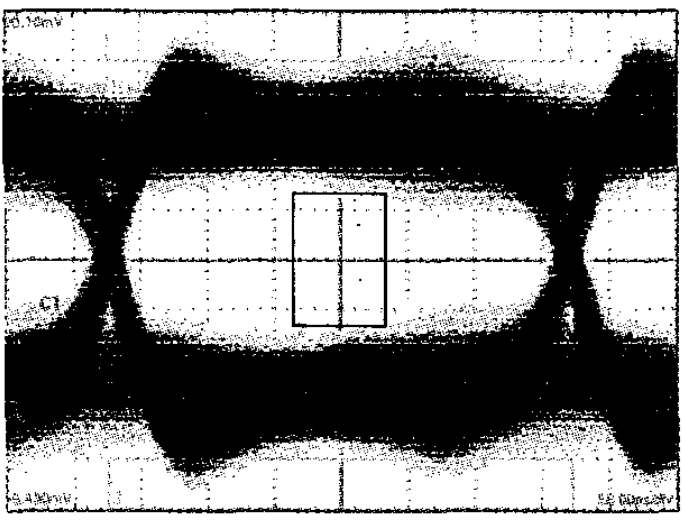

(a) Eye diagram at $2.5 \mathrm{~Gb} / \mathrm{s}$

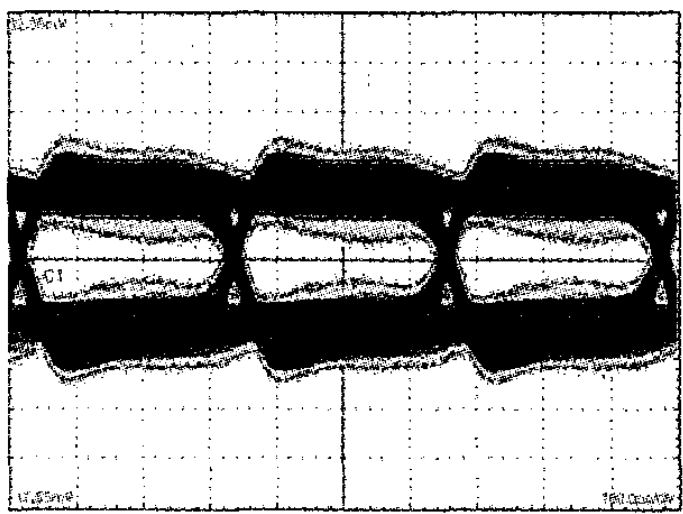

(b) Eye diagram at $3.125 \mathrm{~Gb} / \mathrm{s}$

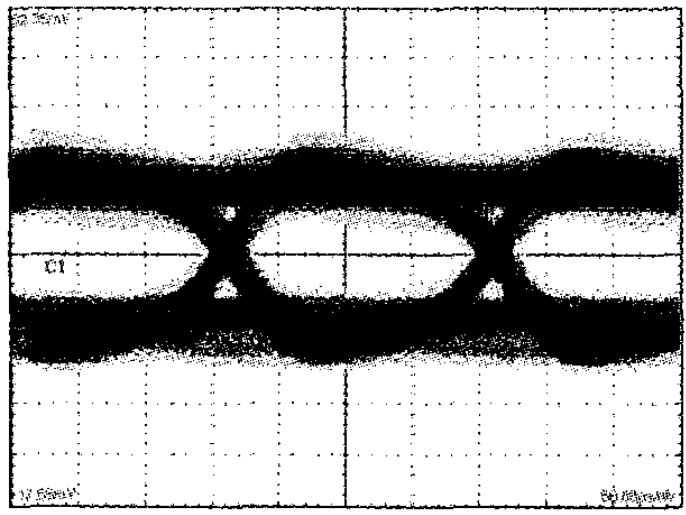

(c) Eye diagram at $5 \mathrm{~Gb} / \mathrm{s}$

Fig. 5 Measured eye diagrams

\section{REFERENCE}

[1] J. J. Morikuni and S. M. Kang, "An analysis of inductive peaking in photoreceiver design," Journal of Lightwave Technology, vol. 10, pp. $1426-1437$, Oct. 1992.

[2] A. A. Abidi, "On the choice of optimum FET size in wide-band transimpedance amplifiers," Journal of Lightwave Technology, vol. 6, pp. 64 -66, Jan. 1988.

[3] S. Galal and B. Razavi, "10Gb/s Limiting Amplifier and Laser/Modulator Driver in 0.18um CMOS Technology," Dig. Tech. Papers ISSCC, pp.188-189, Feb. 2003.

[4] C. C. Tang, C. H. Wu, and S. I. Liu, "Miniature 3D inductors in standard CMOS process," IEEE Journal of Solid-State Circuits, vol. 37, pp. 471-480, April 2002.

[5] J. M. Khoury, "On the design of constant settling time AGC circuits," IEEE Transactions on Circuits and Systems II: Analog and Digital Signal Processing, vol. 45, pp. 283 -294, Mar 1998.

[6] M. Aiki, "Low-noise Optical Receiver for High-speed Optical Transmission," IEEE Trans. Electron Dev., vol. ED-32, pp. 2693-2698, Dec. 1985.

[7] H. H. Kim, S. Chandrasekhar, C. A. Burrus, and J. Bauman, "A Si BiCMOS transimpedance amplifier for $10-\mathrm{Gb} / \mathrm{s}$ SONET receiver," IEEE Journal of Solid-State Circuits, vol. 36, pp. 769 -776 , May 2001 . 